\title{
Real-time and pre-burst optical data for GRBs
}

\author{
R. Hudec ${ }^{1}$, Z. Ceplecha ${ }^{1}$, P. Spurný ${ }^{1}$, J. Florián ${ }^{1}$, A. Kolár ${ }^{1}$, J. Boček ${ }^{1}$, J. Borovička ${ }^{1}$, and P. Kroll ${ }^{2}$ \\ 1 Astronomical Institute, CZ-251 65 Ondřejov, Czech Republic \\ 2 Sonneberg Observatory, Sonneberg, Germany
}

Received January 21; accepted May 10, 1999

\begin{abstract}
The photographic sky patrols still represent a unique database for investigations of positions of GRBs at the times just before, during and after the gamma-ray triggers. We report on the use of two such patrols for these purposes.
\end{abstract}

Key words: gamma-ray bursts — optical transients — sky monitoring

\section{Introduction}

The low energy emission of Gamma Ray Bursts (GRBs) has been confirmed by recent detections of Optical Afterglows (OA) of GRBs observed by BeppoSAX. The optical detections of GRBs starts at $3 \mathrm{hrs}$ after the GRB occurrence in the best case. This is roughly the technical limit of BeppoSAX GRB detection and distribution method: no information can be sent to ground based observers prior this limit. This means that no deeper optical data (mag 12 or better) are available for GRBs for times before and during the gamma ray events as well as immediately (less than $3 \mathrm{hrs}$ ) after for precisely positioned BeppoSAX triggers. The above mentioned technical limit also means that for these triggers, the real-time optical data can be provided only by sky patrol experiments.

Two such patrols are operated in the world, namely the variable star patrol at the Sonneberg Observatory and the meteor patrol at the Ondřejov Observatory. Here we report on the preliminary results of the investigation of Sonneberg and Ondřejov sky patrol plates for GRBs including the precisely positioned GRBs (by CGRO Comptel and by BeppoSAX) and discuss the importance of such real-time and pre-burst optical data for GRBs.

Send offprint requests to: R. Hudec

\section{Data}

The Sonneberg sky patrol is operated every clear night by an array of twelve $30 \times 30 \mathrm{deg}^{2}$ cameras with photographic records. Blue and red emulsions are in use and their mean limiting magnitudes amount to about mag 14.5.

The Ondřejov sky patrol is operated at 11 ground stations equipped by 2 guided and 11 fixed photographic red sensitive cameras reaching lim magnitudes between 4 (for fixed cameras) and 11 (for guided cameras) over fields of view of $180 \mathrm{deg}$ diameter. The recent improvements will result in the sensitivity increase to mag 6 for fixed and to magnitude $12-13$ for guided cameras in the best cases.

\subsection{The Ondřejov sky patrol parameters}

- Optics: Fish-Eye Objective F-Distagon 3.5/30.

- Detector: Planfilm FOMAPAN 400 ASA or 100 ASA (panchromatic emulsion) $90 \times 120 \mathrm{~mm}$, sky diameter $80 \mathrm{~mm}$.

- Typical exposure time: 3 hrs for guided cameras, whole night for fixed cameras.

- 2 stations equipped with guided and fixed cameras.

- 9 stations equipped with fixed cameras.

- Sensitivity for brief $1 \mathrm{~s}$ triggers $2-3 \mathrm{mag}$, for stars up to mag 11; after improvements in 1998 mag $4-6$ for 1 s flashes and mag $12-13$ for stars.

- Response limited to the red light above $400 \mathrm{~nm}$.

- Preferences: (1) Large sky coverage (full visible hemisphere), (2) Large fraction of observation time: 2400 to $6000 \mathrm{srh}$ for one station/year, (3) Multiplicity of data to eliminate background triggers easily, (4) Classification of detected triggers by parallax, (5) Simultaneous and pre-burst optical data (limits) for GRBs.

Both data sets have been examined for observations covering the GRB positions at times during as well as immediately before and after the triggers (within 2 days). 


\section{Results}

\subsection{General results, Ondřejov observatory}

No optical emission above mag 5 (1 s duration assumed) or mag 13 (full exposure time) or Lg/Lo $>100 \ldots 300$ has been detected for a few GRBs. The faintest limit (320) exists for GRB 830313 (Hudec 1993). No optical emission above magnitudes $0 \ldots 3$ (1 s duration assumed) or $4-11$ (full exposure time) or $\mathrm{Lg} / \mathrm{Lo}>0.1 \ldots 10$ has been detected for many (95) GRBs.

Optical transient (OT) has been detected on the plate taken $\sim 7 \mathrm{~h}$ after the GRB 790929 inside its error box (Borovička et al. 1992)

\subsection{Examples of BeppoSAX related results, Ondřejov observatory}

\subsubsection{GRB 960720 11:37 UT}

- GRB at daytime, plates available for night before and after:

- 960719/20 7 plates 20:40 - 01:27 UT lim mag 4-11 for stars,

- 960720/21 8 plates 20:45 - 01:30 UT lim mag $4-11$ for stars,

- 960930 BVR CCD 18:35 UT lim mag 20.

- Results: no optical activity 10 h before GRB limit 11 and $9 \mathrm{~h}$ after GRB limit 11.

\subsubsection{GRB 970111 09:44 UT}

- GRB at daytime, plates available for the night after:

- 970111/12 1 plate 16:45 - 20:15 UT lim mag 4,

- 970208 03:25 UT BVR CCD lim mag 20,

- Results: no optical activity $7 \mathrm{~h}$ after GRB limit 4.

\subsubsection{GRB 970228 02:28 UT}

- GRB at nighttime but below the local horizon at the time of event, plates available for the night of GRB:

- 970227/28 2 plates 17:56 - 21:58 UT lim mag 4,

- 970227/28 9 plates 00:50 - 04:32 UT lim mag 4-10 but GRB below horizon,

- 970228/0301 23 plates 17:56 - 01:46 lim mag 4-11,

- 970303 BVR CCD 19:30 UT lim mag 20.

- Results: No optical activity 4.5 h before GRB lim mag 4 and $13.5 \mathrm{~h}$ after GRB lim mag 10.

\subsection{Examples Sonneberg results, COMPTEL GRBs}

17 time-correlated photographic plates with limiting magnitudes around 14.5 have been investigated for the following three COMPTEL GRBs.
For GRB 910709 at UT 11.56, no optical activity except variable star Y Dra mentioned later has been found on patrol plates taken $11 \mathrm{~h}$ before and $35 \mathrm{~h}$ after. For GRB 940708 at UT 20.70, no optical activity has been revealed on plates taken $21 \mathrm{~h}$ before, $3 \mathrm{~h}$ after and $27 \mathrm{~h}$ after. For GRB 940301 at 20.18 UT, no optical activity has been found on the patrol plates taken $24 \mathrm{~h}$ before and $71 \mathrm{~h}$ after.

Conclusions: No new and/or variable optical objects with amplitudes more than 0.4 mag have been found except for GRB 910709. In this case, a bright object with maximum close to the GRB date and the fading has been revealed and identified with a Mira type variable star Y Dra (variable between mag 6.24 and 15.0). Although the objects are probably not related, this is an another example of background trigger with analogous appearance to real optical afterglows of GRBs.

From the number of 20 BeppoSAX/RXTE GRBs listed, a subset of $8 \mathrm{GRBs}$ is covered in location and in time (criterion: \pm 7 days) by the Sonneberg Sky Patrol (altogether 67 plates, evaluation is still in progress).

\section{Optical transients versus optical afterglows}

The recently detected optical transients (OTs) related to GRBs are generally considered as optical afterglows while the direct optical emission of bursts remains to be unknown. The data presented here hence represent valuable limits for direct optical luminosities of GRBs in question: the direct optical emission seems not to exceed $V$ magnitudes $7-10$.

\section{Conclusions}

Despite the recent instrumental developments, the photographic patrol data still represent the unique databases for real-time (simultaneous and pre-bursts) optical observations of GRBs. The recently operated alert systems can provide rapid follow up optical data (with time delays of order of tens of seconds) for less precisely localized CGRO BATSE triggers but their magnitude limits in this case are generally not substantially better (since they have to cover large FOVs) than those of photographic sky patrols and further, they will never be able to provide data for times before and during burst triggers. The improved sky patrols can yield valuable real-time and pre-burst optical data in the magnitude range $12-15$.

Acknowledgements. The work has been supported by the Projects of the Czech Ministry for Education and Youth, Nos. ES02 and ES036.

\section{References}

Borovička J., et al., 1992, A\&A 258, 379

Hudec R., 1993, Astro. Lett. Comm. 28, 359 